\title{
CONCERNING THE HAHN-BANACH THEOREM 1
}

\author{
JOHN A. R. HOLBROOK
}

\begin{abstract}
We establish an intrinsic characterization for those normed spaces having the extension property that applies equally to spaces with real, complex, or quaternionic scalars. Nachbin's characterization for real spaces via the binary intersection property follows as a special case. The method also yields a proof of the Hahn-Banach theorem that does not depend on the choice of scalar field.
\end{abstract}

The original motivation for this work was dissatisfaction with the traditional proof of the Hahn-Banach theorem for complex normed linear spaces. Several years ago we found an alternate argument that applies equally to real, complex, or quaternionic scalars, and noted that the technique of our proof suggests an intrinsic characterization of those normed spaces with the extension property (see Theorems 3 and 5). The referee of the present write-up has pointed out that similar results appear in the preprint [5] by O. Hustad.

We recall that the Hahn-Banach extension theorem for real spaces dates from papers by H. Hahn [3] in 1927 and S. Banach [1] in 1929, while the familiar trick deriving the theorem for other scalars by reduction to the real case was not forthcoming until 1938: H. F. Bohnenblust and A. Sobczyk [2] (complex scalars); G. A. Soukhomlinoff [9] (complex or quaternionic scalars). The number of intervening years indicates that this trick, simple though it may be, is neither natural nor inevitable. In fact, we may base the extension theorem on the following lemma, establishing a simple property shared by the three scalar fields used with normed linear spaces: the reals $R$, the complex numbers $\mathbf{C}$, and the quaternions $\mathbf{Q}$. In preparation we merely note that each of these scalar fields $F$ has a conjugation (indicated as usual by a bar) such that $z \bar{z}=|z|^{2}$, and that the distance $|z-w|$ in $F$ is Euclidean.

Received by the editors May 10, 1971 and, in revised form, November 7, 1973 and May 3, 1974.

AMS (MOS) subject classifications (1970). Primary 46B99.

Key words and phrases. Hahn-Banach theorem, complex normed spaces, extension property.

1 This work was supported in part by the National Research Council of Canada under Grant No. A8746, and in part by USAF Grant AFOSR 1322-67. 
Lemma 1. Each scalar field $F$ has the following "intersection property"': if $c_{k} \in F$ and $r_{k}>0$, the balls

$$
B_{r_{k}}\left(c_{k}\right)=\left\{z \in F:\left|z-c_{k}\right| \leq r_{k}\right\} \quad(k=1,2, \cdots, n)
$$

have nonempty intersection provided $\left|\Sigma_{1}^{n} z_{k} c_{k}\right| \leq \Sigma_{1}^{n}\left|z_{k}\right| r_{k}$ for any $z_{k} \in F$ such that $\Sigma_{1}^{n} z_{k}=0$.

Proof. Let $m$ be the minimum value of the function $f$ defined on $F$ by

$$
f(z)=\max _{1 \leq k \leq n} \frac{\left|z-c_{k}\right|}{r_{k}},
$$

and let $\beta \in F$ be a point at which this minimum is attained. We may assume that $\left|\beta-c_{k}\right| / r_{k}=m$ for $1 \leq k \leq p$, while $\left|\beta-c_{k}\right| / r_{k}<m$ for $p<k \leq n$. Now $\beta$ must lie in the convex hull $K$ of $c_{1}, c_{2}, \ldots, c_{p}$. Otherwise, if $\beta_{0}$ is the point in $K$ closest to $\beta$, then the dot product $\left(\beta-\beta_{0}\right) \cdot\left(c_{k}-\beta_{0}\right)$ is nonpositive for $1 \leq k \leq p$. Hence, for any $z$ between $\beta$ and $\beta_{0}$ and $1 \leq k \leq p$, $\left|z-c_{k}\right|<\left|\beta-c_{k}\right|$ so that $\left|z-c_{k}\right| / r_{k}<m$. Since this last inequality holds also for $p<k \leq n$ when $z$ is close to $\beta$, we would have $f(z)<m$, a contradiction.

We have, then, $\beta=\Sigma_{1}^{P} \lambda_{k} c_{k}$, where $\lambda_{k} \geq 0$ and $\Sigma_{1}^{P} \lambda_{k}=1$. Set $z_{k}=$ $\lambda_{k} \overline{\left(\beta-c_{k}\right)}$ and note that $\Sigma_{1}^{P} z_{k}=0$, and that $z_{k}\left(\beta-c_{k}\right)=\lambda_{k}\left|\beta-c_{k}\right|^{2} \geq 0$. Hence,

$$
m \sum_{1}^{P}\left|z_{k}\right| r_{k}=\sum_{1}^{P}\left|z_{k}\right|\left|\beta-c_{k}\right|=\left|\sum_{1}^{P} z_{k}\left(\beta-c_{k}\right)\right|=\left|\sum_{1}^{P} z_{k} c_{k}\right| \leq \sum_{1}^{P}\left|z_{k}\right| r_{k},
$$

so that $m \leq 1$. Q.E.D.

Theorem 2. If $S$ is a subspace of the normed linear space $(X,\|\cdot\|)$ with scalars $F$, and $\phi: S \rightarrow F$ is a (linear) functional such that $(\forall s \in S$ ) $|\phi(s)| \leq\|s\|$, then there exists a functional $\tilde{\phi}: X \rightarrow F$ extending $\phi$ and such that $(\forall x \in X)|\tilde{\phi}(x)| \leq\|x\|$.

Proof. By the usual induction (possibly transfinite, if $X / S$ is big), we reduce to the "one-step extension problem" where $X$ is spanned by $S$ and a single additional vector $u$. We must simply find a value $a \in F$ for $\widetilde{\phi}(u)$, and the condition on $\alpha$ is easily seen to be the following: $(\forall s \in S)|\alpha-\phi(s)|$ $\leq\|u-s\|$. In the notation of Lemma 1, we must show that the balls $\left\{B \|_{u-s \|}(\phi(s)): s \in S\right\}$ have nonempty intersection. Since each ball is compact we need only show that any finite collection of the balls has nonempty intersection. By Lemma 1 , it suffices to note that, whenever $s_{k} \in S, z_{k} \in F$, and $\Sigma_{1}^{n} z_{k}=0$, 
$\left|\sum_{1}^{n} z_{k} \phi\left(s_{k}\right)\right|=\left|\phi\left(\sum_{1}^{n} z_{k} s_{k}\right)\right| \leq\left\|\sum_{1}^{n} z_{k} s_{k}\right\|=\left\|\sum_{1}^{n} z_{k}\left(s_{k}-u\right)\right\| \leq \sum_{1}^{n}\left|z_{k}\right|\left\|u-s_{k}\right\|$. Q.E.D.

In the study of extension theorems for linear transformations more general than functionals, the following notion has received considerable attention: a normed linear space $(Y,\|\cdot\|$ ) (with scalar field $F$ ) is said to have the extension property (EP) provided that, for any normed linear space $(X,\|\cdot\|)$ (with scalars $F$ ), subspace $S$ of $X$, and linear transformation $T$ : $S \rightarrow Y$ such that $(\forall s \in S)\|T(s)\| \leq\|s\|$, there exists a linear extension $\tilde{T}$ : $X \rightarrow Y$ such that $(\forall x \in X)\|\widetilde{T}(x)\| \leq\|x\|$. For example, Theorem 2 says that any one-dimensional $Y$ (which is thus isomorphic to $F$ ) has the (EP).

Several characterizations of those spaces $Y$ having the (EP) are available. It is known, for example, that $Y$ has the (EP) if, and only if, $Y$ has the projection property: there is a contractive projection onto $Y$ from any normed linear space $Z$ containing $Y$ as a subspace. In another direction, J. L. Kelley [6] and M. Hasumi [4] have shown that the spaces $Y$ with the (EP) are precisely those isomorphic to $C(H)$, the space of continuous scalar functions on some Stonian space $H$. These characterizations are not, however, intrinsic; that is, they are not expressed simply in terms of the geometry of the space $Y$.

L. Nachbin [8] gives us an elegant intrinsic description of those real normed spaces $Y$ having the (EP). Here, and later, $B$ always denotes a collection of balls in $Y$; that is, each $B \in \mathfrak{B}$ has the form

$$
B=B_{r}(c)=\{y \in Y:\|y-c\| \leq r\},
$$

for some $c \in Y$ and $r \geq 0$. Nachbin shows that, for $(Y,\|\cdot\|)$ with scalars $F=\mathbf{R}, Y$ has the (EP) if, and only if, $Y$ has the binary intersection property:

$$
\left(\forall B_{1}, B_{2} \in \mathscr{B}\right) B_{1} \cap B_{2} \neq \varnothing \Rightarrow \bigcap \Re \neq \varnothing
$$

For further work on the case $F=\mathbf{R}$, the reader might consult the memoir [7] by J. Lindenstrauss. The analysis of the Hahn-Banach theorem that we have presented in Lemma 1 and Theorem 2 suggests an intrinsic characterization of the (EP) which is valid for any of the scalar fields $F=\mathbf{R}, \mathbf{C}$, or Q. Theorems 3 and 5, below, state precise results of this nature. In Corollary 6 we will see that Nachbin's result follows naturally upon adding the assumption that $F=\mathbf{R}$.

We shall say that a normed linear space $Y$, with scalars $F$, has the 
intersection property (IP) provided it mimics the behavior of the scalar fields $F$ established in Lemma 1 . More precisely, we say $Y$ has (IP) if any collection $B$ of balls in $Y$ has nonempty intersection $(\bigcap \Re \neq \varnothing)$ whenever

$$
B_{r_{k}}\left(c_{k}\right) \in \mathcal{B}, z_{k} \in F \text {, and } \sum_{1}^{n} z_{k}=0 \Rightarrow\left\|\sum_{1}^{n} z_{k} c_{k}\right\| \leq \sum_{1}^{n}\left|z_{k}\right| r_{k} .
$$

We remark that it is a simple matter to show that $(\bigcap \Re \neq \varnothing) \Rightarrow(1)$, for any normed space $Y$.

Theorem 3. Let $(Y,\|\cdot\|)$ be a normed linear space with scalars $F$. Then $Y$ has the extension property $(E P)$ if, and only if, it has the intersection property (IP).

Proof. To establish the (EP) for $(Y,\|\cdot\|)$ we consider a linear transformation $T: S \rightarrow Y$ such that $(\forall s \in S)\|T(s)\| \leq\|s\|$, and, following the model of the proof of Theorem 2, we must simply show that $\bigcap \Re \neq \varnothing$ where

$$
\mathfrak{B}=\left\{B_{\|u-s\|}(T(s)): s \in S\right\} .
$$

Since we are assuming (IP), we need only verify (1) for this $B_{\text {; but if }} \Sigma_{k}$ $=0$ and $s_{k} \in S$,

$$
\begin{aligned}
\left\|\sum_{k} z_{k} T\left(s_{k}\right)\right\| & =\left\|T\left(\sum_{k} z_{k} s_{k}\right)\right\| \leq\left\|\sum_{k} z_{k} s_{k}\right\| \\
& =\left\|\sum_{k} z_{k}\left(s_{k}-u\right)\right\| \leq \sum_{k}\left|z_{k}\right|\left\|u-s_{k}\right\| \cdot
\end{aligned}
$$

On the other hand, suppose $Y$ has the (EP) and that $\mathscr{B}$ satisfies (1). Consider the vector space $X$ over $F$ defined by $X=\left\{\Sigma_{k} z_{k} c_{k}: z_{k} \in F, c_{k} \epsilon\right.$ $\mathcal{C}\}$, where $\mathcal{C}=\left\{c \in Y: B_{r}(c) \in \mathscr{B}\right.$ for some $\left.r \geq 0\right\}$. Introduce the seminorm $\|\cdot\|_{0}$ on $X$ as follows:

$$
\|x\|_{0}=\inf \left\{\sum_{k}\left|z_{k}\right| r_{k}: x=\sum_{k} z_{k} c_{k} \text { and } B_{r_{k}}\left(c_{k}\right) \in \mathcal{B}\right\} \text {, }
$$

and identify elements of $X$ at distance 0 with respect to the seminorm. The set $S=\left\{\Sigma_{k} z_{k} c_{k}: z_{k} \in F, \Sigma_{k} z_{k}=0, c_{k} \in \mathcal{C}\right\}$ is a subspace of $X$ and (1) simply assures that $(\forall s \in S)\|s\| \leq\|s\|_{0}$. Hence the identity map $T$ on $S$ is contractive, and by the (EP) $T$ has a contractive linear extension $\tilde{T}$ to $X$. Note that, for any pair $c, c^{\prime} \in \mathcal{C}, c-c^{\prime} \in S$, so that

$$
\tilde{T}(c)-\tilde{T}\left(c^{\prime}\right)=\tilde{T}\left(c-c^{\prime}\right)=T\left(c-c^{\prime}\right)=c-c^{\prime} .
$$


Let $y$ denote the element of $Y$ such that $(\forall c \in \mathcal{C}) y=c-\widetilde{T}(c)$. It follows that

$$
B_{r}(c) \in \mathscr{B} \Rightarrow\|y-c\|=\|\tilde{T}(c)\| \leq\|c\|_{0} \leq 1 \cdot r,
$$

so that $y \in \bigcap \Re$. Q.E.D.

Lemma 4. In any normed linear space $Y$ with scalars $F,(1)$ is equivalent to the following statement concerning $\mathfrak{B}$ :

(2) $z_{k} \in F,\left|z_{k}\right|=1, \sum_{k} z_{k}=0$, and $B_{r_{k}}\left(c_{k}\right) \in \mathfrak{B} \Rightarrow\left\|\sum_{k} z_{k} c_{k}\right\| \leq \sum_{k} r_{k}$.

Proof. Certainly ( 1$) \Rightarrow(2)$. On the other hand, (1) follows by continuity once it is known for $z_{k}$ which are "commensurable" in the sense that, for some $\epsilon>0$ and integers $n_{k},\left|z_{k}\right|=n_{k} \epsilon$. In this case, let $w_{k}=z_{k} / n_{k} \epsilon$, and observe that $\left|w_{k}\right|=1, \Sigma_{k} n_{k} w_{k}=(1 / \epsilon) \Sigma_{k} z_{k}=0$. Thus (2) ensures that $\left\|\Sigma_{k} n_{k} w_{k} c_{k}\right\| \leq \Sigma_{k} n_{k} r_{k}$, so that

$$
\left\|\sum_{k} z_{k} c_{k}\right\|=\epsilon\left\|\sum_{k} n_{k} w_{k} c_{k}\right\| \leq \sum_{k} \epsilon n_{k} r_{k}=\sum_{k}\left|z_{k}\right| r_{k} .
$$

Lemma 4 allows us to replace Theorem 3 by the following variant, from which Nachbin's theorem is conveniently derived as a special case.

Theorem 5. Let $(Y,\|\cdot\|)$ be a normed linear space with scalars $F$. Then $Y$ has the (EP) if, and only if, it has the intersection property $\left(\mathrm{IP}_{1}\right)$

$$
\bigcap \mathfrak{B} \neq \varnothing \quad \text { whenever } B \text { satisfies (2). }
$$

Corollary 6 (Nachbin). A real normed linear space has the (EP) if, and only if, it has the binary intersection property (2-IP).

Proof. We simply check that, when $F=\mathbf{R},(2)$ is equivalent to

$$
\left(\forall B_{1}, B_{2} \in \mathscr{B}\right) B_{1} \cap B_{2} \neq \varnothing \text {. }
$$

Setting $z_{1}=1, z_{2}=-1$ in (2) we obtain $\left\|c_{1}-c_{2}\right\| \leq r_{1}+r_{2}$, which simply means that $B_{r_{1}}\left(c_{1}\right) \cap B_{r_{2}}\left(c_{2}\right) \neq \varnothing$. Thus (for any $F$ ) (2) $\Rightarrow(3)$, and hence $(2-\mathrm{IP}) \Rightarrow(\mathrm{EP})^{1}$. On the other hand, if $\Sigma_{z_{k}}=0,\left|z_{k}\right|=1$, and $z_{k} \in \mathbf{R}$, we may clearly assume that $z_{1}=1, z_{2}=-1, z_{3}=1, \ldots, z_{2 p}=-1$. Assuming (3), we have $\left\|c_{2 j-1}-c_{2 j}\right\| \leq r_{2 j-1}+r_{2 j}$, and hence

$$
\begin{aligned}
\left\|\sum z_{k} c_{k}\right\| & =\left\|\sum_{j}\left(c_{2 j-1}-c_{2 j}\right)\right\| \\
& \leq \sum_{j}\left\|c_{2 j-1}-c_{2 j}\right\| \leq \sum_{j}\left(r_{2 j-1}+r_{2 j}\right)=\sum_{k} r_{k} \text {. Q.E.D. }
\end{aligned}
$$


Remark. While $\mathbf{R}$ has the binary intersection property (2-IP), $\mathbf{C}$ has the ternary intersection property ( $3-\mathrm{IP}): \bigcap \Re \neq \varnothing$ provided that

$$
\left(\forall B_{1}, B_{2}, B_{3} \in \Re\right) B_{1} \cap B_{2} \cap B_{3} \neq \varnothing .
$$

This is a special case of Helly's theorem on convex sets in $\mathbf{R}^{n}$. In view of Nachbin's theorem, it is natural to ask whether the complex spaces with the (EP) can be characterized by (3-IP). It is not hard to show that (EP) $\Rightarrow$ (3-IP) by imbedding $Y$ as a subspace of the bounded $\mathrm{C}$-valued functions on $U^{*}=$ the unit ball in the dual space $Y^{*}$. We do not know whether $(3-\mathrm{IP}) \Rightarrow(\mathrm{EP})$ when $F=\mathbf{C}$, but in any case we cannot proceed as in our proof of Corollary 6, since examples show that (4) need not follow from (2).

\section{REFERENCES}

1. S. Banach, Sur les fonctionnelles linéaires. I, II, Studia Math. 1 (1929), 211-216, 223-239.

2. H. F. Bohnenblust and A. Sobczyk, Extensions of functionals on complex linear spaces, Bull. Amer. Math. Soc. 44 (1938), 91-93.

3. H. Hahn, Über lineare Gleichungssysteme in linearen Räumen, J. Reine Angew. Math. 157 (1927), 214-229.

4. M. Hasumi, The extension property of complex Banach spaces, Tôhoku Math. J. (2) 10 (1958), 135-142. MR 20 \# 7209.

5. O. Hustad, Intersection properties of balls in complex Banach spaces whose duals are $L^{1}$-spaces, Preprint Series, Univ. of Oslo.

6. J. L. Kelley, Banach spaces with the extension property, Trans. Amer. Math. Soc. 72 (1952), 323-326. MR 13, 659.

7. J. Lindenstrauss, Extension of compact operators, Mem. Amer. Math. Soc. No. 48 (1964). MR 31 \# 3828.

8. L. Nachbin, A theorem of the Hahn-Banach type for linear transformations, Trans. Amer. Math. Soc. 68 (1950), 28-46. MR 11, 369.

9. G. A. Soukhomlinoff, Über Fortsetzung von linearen Funktionalen in linearen komplexen Räumen und linearen Quaternioneräumen, Mat. Sb. 3 (45) (1938), 353358. CANADA

DEP ARTMENT OF MATHEMATICS, UNIVERSITY OF GUELPH, GUELPH, ONT ARIO, 